Vol. 19(1):40-45, abril - julio 2016

\title{
Investigación en el pregrado de Medicina: una experiencia universitaria formativa
}

\author{
Research in the Medicine undergraduate program: \\ an educational university experience \\ Pesquisa em Graduação de Medicina: uma \\ experiência universidataria educacional
}

\author{
César Ramón Romero-Leguizamón, MD. * \\ Juan Felipe Quintero-Moreno. ** \\ Andrés Mauricio García-Cuevas. ** \\ Cristhian David Arroyave-Durán. **
}

\section{Resumen}

Introducción: En nuestro país existe una figura denominada semillero de investigación como una herramienta de promoción de la formación integral de los estudiantes, que tiene el objetivo de promover nuevo talento en áreas investigativas que permita la generación de nuevo conocimiento. En consecuencia, el Departamento Administrativo de Ciencia, Tecnología e Innovación (Colciencias) y las universidades del país han creado múltiples estrategias para potenciar habilidades investigativas en sus estudiantes. Objetivo: Reflexionar acerca de la importancia de la investigación en el pregrado mediante la estrategia pedagógica de los semilleros de investigación en áreas de la salud. Reflexión: Esta estrategia permite mejorar la formación del estudiante y hacerlo competitivo en el contexto nacional e internacional. Sin embargo, estos esfuerzos no son suficientes pues se carece de recursos económicos, planes curriculares flexibles y docentes capacitados en investigación; todos estos factores, sumados al insuficiente interés de los estudiantes, convierten la tarea en un objetivo difícil de lograr. Conclusión: Los semilleros de investigación forman personas en trabajo colaborativo y desarrollan destrezas que permiten la formación integral de los estudiantes. [Romero CR, Quintero JF, Arroyave CD,
García AM. Investigación en el pregrado de Medicina: una experiencia universitaria formativa. MedUNAB 2016; 19 (1): 40-45]

Palabras clave: Estudiantes de Medicina; Educación Médica; Investigación; Educación; Aprendizaje; Colombia.

\begin{abstract}
Introduction: In our country there is a figure called hotbed of research as a tool to encourage the comprehensive training of students, which aims to promote new talent in investigative areas that allow the generation of new knowledge. Additionally, the Administrative Department of Science, Technology and Innovation (Colciencias) and Colombian universities have created multiple strategies to enhance research skills in their students. Objective: To reflect on the importance of research in undergraduate programs by using the hotbed of research teaching strategy in health areas. Reflection: This strategy allows improving students' training and making it more competitive at national and international level. However, these efforts are not enough because it lacks sufficient financial resources, flexible curricula and teachers trained in research; all these factors, along with deficient student interest, transform the task into a difficult goal to
\end{abstract}

\footnotetext{
* Médico general, Universidad Tecnológica de Pereira, Colombia.

** Estudiante de Medicina, Semillero de Fisiología Aplicada y Neurociencias (SEFAN), Facultad de Ciencias de la Salud, Universidad Tecnológica de Pereira, Colombia.
}

Autor de correspondencias: César Ramón Romero Leguizamón, dirección de correspondencia: calle 27 \# 102 Universidad Tecnológica de Pereira, Pereira, Colombia. Correo electrónico: crromero@utp.edu.co. 
achieve. Conclusion: Hotbeds of research educate people in collaborative work, and also develop skills that allow the comprehensive training of students. [Romero CR, Quintero JF, Arroyave CD, García AM. Research for Medical undergraduate students: a university training experience. MedUNAB 2016; 19 (1): 40-45]

Key words: Medical Students; Medical Education; Research; Education; Learning; Colombia.

\section{Resumo}

Introdução: Na Colômbia existe uma tipo de grupo de estudantes chamado "semillero=sementeira" de pesquisa como uma ferramenta para promover a formação integral dos alunos, que visa promover novos talentos nas áreas da pesquisa com o objetivo de gerar novos conhecimentos. Além disso, o Departamento Administrativo de Ciência, Tecnologia e Inovação (Colciencias) e as universidades do país criaram várias estratégias para melhorar as habilidades de pesquisa em seus alunos. Objetivo: refletir sobre a importância da pesquisa com estudantes na graduação como uma estratégia pedagógica do ensino superior, no campo da saúde. Reflexão: Esta estratégia permite melhorar a formação dos alunos e torná-la competitiva a nível nacional e internacional. No entanto, estes esforços não são suficientes porque não conta com recursos financeiros suficientes, currículos flexíveis e professores treinados em pesquisa; todos esses fatores, mais o insuficiente interesse dos alunos, fazem com que o objetivo seja difícil de alcançar. Conclusão: Os grupos de pesquisa com estudantes formam as pessoas no trabalho colaborativo e desenvolvem habilidades que permitem a formação integral dos alunos. [Romero CR, Quintero JF, García AM, Arroyave CD. Pesquisa em Graduação de Medicina: uma experiência universidataria educacional. MedUNAB 2016; 19(1): 40-45]

Palavras chave: Estudantes de Medicina; Educação Médica; Pesquisa; Educação; Aprendizagem; Colômbia.

"Un cientifico en su laboratorio no es solo un técnico: es también un niño colocado ante fenómenos naturales que le impresionan como en un cuento de hadas".

Marie Curie

\section{Introducción}

En el hombre son innatas las facultades que propician el entendimiento de los fenómenos que suceden a su alrededor; tales facultades se pueden perfeccionar $\mathrm{y}$ modelar a través de un proceso de enseñanza, que involucre las capacidades para indagar, proponer, debatir y producir nuevo conocimiento(1). Los espacios ideales, creados para gestary desarrollar estas habilidades en el ámbito educativo, son los denominados (por su analogía con el mundo vegetal) semilleros de investigación, espacios que buscan ser propicios para la formación integral del estudiante, pues es allí en donde se deben dar gran parte de las herramientas que necesita el talento juvenil(2).

La motivación para escribir esta reflexión surge a partir del crecimiento personal y colectivo de los autores como "semillas" en investigación, además por la preocupante poca visibilización y financiación de los proyectos que allí se idean, plantean y estructuran. En Colombia esta estrategia no ha sido aún evaluada de forma objetiva, desconociéndose la magnitud e impacto de los semilleros de investigación. Esta reflexión invita a discernir diferentes aspectos como: la importancia de la investigación estudiantil, su efecto sobre la formación integral, lo que implica dentro del proceso formativo del estudiante de medicina, el relevo generacional en el camino de la ciencia, la idoneidad de los tutores y finalmente la magnitud de los semilleros como un espacio sensibilizador hacia la formación científica.

\section{La importancia de la investigación estudiantil en Medicina}

La investigación se fundamenta en diferentes elementos que son piezas de un proceso de retroalimentación y de constante cambio (3), los cuales se convierten en herramientas de aplicabilidad social y estrategias de enseñanza y aprendizaje fundamentales para el estudiante de medicina(4).

Aprender a investigar es uno de los componentes necesarios para alcanzar la formación integral del estudiante de pregrado y posgrado especialmente para el personal de ciencias de la salud (5), pues durante este proceso se desarrollan diferentes tipos de destrezas para el ejercicio reflexivo, como la lectura crítica, la adecuada búsqueda y selección de información, la redacción de manuscritos científicos $(6,7)$, entre otras. además, el contacto con el ambiente investigativo desde el pregrado favorece el interés del estudiante por querer continuar estudios de posgrado y contribuir a la resolución de problemas por medio del desarrollo de ideas y proyectos de investigación $(8,9)$.

Hace dos décadas, en la facultad de medicina de la Universidad de Stanford, se implementaron las llamadas cátedras de investigación, ya que los programas de formación no eran suficientes para cumplir con el requerimiento de investigadores biomédicos, determinado por el poco interés mostrado por parte de los estudiantes para dedicarse a esa 
área; eso sin contar con las dificultades planteadas por la consecución de la financiación para investigar. Para fomentar este interés en los estudiantes, acciones como la flexibilidad curricular, los incentivos financieros, las ayudantías de investigación y los reconocimientos especiales para los estudiantes que hacen investigación, fueron implementadas satisfactoriamente (10). Por otra parte, la vinculación de los profesionales médicos a la ciencia básica es algo fundamental para que la investigación biomédica avance y así se pueda generar un impacto en la atención en salud, por la integración básico-clínica, que orienta los procesos investigativos hacia la implementación de nuevas terapias que den solución a los problemas de salud pertinentes (11).

\section{La investigación en la formación integral del estudiante}

Por lo general, el estudiante tiene su primer contacto con este proceso cuando cursa un módulo curricular de "metodología de la investigación" en los semestres iniciales de su carrera, el cual se supone debe brindar herramientas para acceder a la información científica, analizarla y discernir sobre ella. Desafortunadamente, los docentes encargados de estos módulos no siempre poseen el perfil adecuado para dictar estas cátedras o carecen de las estrategias pedagógicas idóneas para motivar al estudiante por esta rama del saber $(12,13)$, además, se suele utilizar un modelo tradicional y poco creativo, el cual es rígido y complejo, convirtiéndose en una experiencia tortuosa y tediosa para el estudiante.

Lo anterior, permite inferir que las habilidades para aprender a investigar no se desarrollan en dichas cátedras y podría explicar la falta de interés de los estudiantes de nuestro país por estos temas $(14,15)$. Otro aspecto relevante es el desconocimiento que tiene el estudiante acerca de los beneficios de aprender a investigar, no sólo desde el punto de vista formativo, sino de otros como la exoneración y descuentos en el pago de matrícula, la posibilidad de publicar en una revista científica de un adecuado nivel y una hoja de vida sobresaliente que impacte al momento de presentarse para realizar estudios de posgrado $(8,16)$. Con base en lo anterior se justifica la aparición de los semilleros de investigación como un espacio donde los interesados en desarrollar ideas o propuestas investigativas en áreas afines a su preferencia personal, pueden hallar herramientas y apoyo, tanto académico como financiero, por parte de las instituciones educativas y los entes estatales (14). A pesar de esto, el presupuesto dirigido al fomento investigativo es insuficiente y las universidades tienen que hacer grandes esfuerzos para brindar algún apoyo a los semilleros en el desarrollo de sus propuestos $(9,17)$.

Usualmente, y como puede ser consultado en las diferentes plataformas virtuales de los programas de Medicina del país, la malla curricular de cada de uno de ellos puede resumirse en tres grandes núcleos de objetivos académicos: Ciencias Básicas, Ciencias Clínicas y Salud comunitaria. En ciertas universidades se encuentra un adicional correspondiente a un sistema de electivas que en algunas instituciones se limita a un número reducido de créditos en un semestre aislado, en contraste, con otras donde los estudiantes pueden vincularse durante todo su programa académico a electivas que incluyen áreas de profundización del área Médica hasta la artística y de Ciencias exactas. ¿Cómo flexibilizar lo inflexible? Es la pregunta que se debería formular a los comités curriculares de los programas de Medicina, acaso es imperativo para la formación médica aplicar la mayor cantidad de carga horaria dirigida a saturar al estudiantado, o pueden facilitarse los conceptos básicos de los núcleos de conocimientos y las habilidades de búsqueda de información e interpretación de evidencia científica, mediante un sistema de áreas de profundización asociadas al objetivo del año curricular inmerso en un proceso de desarrollo investigativo, con el fin de estimular los intereses y habilidades individuales de los estudiantes en diversas áreas, generando perfiles que satisfagan las necesidades no solo de desarrollo personal sino los potenciales candidatos a garantizar la transición generacional en los múltiples campos de acción del egresado en Medicina general. Es tiempo de cambiar el viejo adagio de "un mar de conocimientos de un centímetro de profundidad", egresados saturados pero embebidos en conocimientos poco precisos y confusos o egresados con áreas de profundización específicas, pero con criterios fuertes de discernimiento científico-clínico.

\section{Quiénes son los tutores dentro de un semillero de investigación}

En un semillero, el tutor es importante para el desarrollo de los estudiantes, puesto que sus habilidades y su experiencia son el modelo del cual cada estudiante puede nutrirse y desarrollar su perfil investigativo (18). Es necesario recalcar la importancia de los docentes que dirigen las cátedras de investigación o, en este caso, orienten un semillero, puesto que deben ser personas idóneas, flexibles y creativas en su modelo de enseñanza, para que, de esta manera la investigación sea una experiencia agradable que conduzca al estudiante a aprender y disfrutar del acto investigativo, y no un proceso pesado y difícil que sea percibido como una carga '(3).

El espíritu de los semilleros de investigación consiste en vincularse a un grupo de investigación preferiblemente reconocido o que se encuentre en proceso de registro en la plataforma GrupLAC de Colciencias; dicho grupo se encuentra encabezado por un líder de grupo, quien con frecuencia es el tutor a cargo de las "semillas" en formación. Ahora bien, ¿cuáles son las características necesarias para desempeñar esta labor y realmente, consolidar un proceso formativo y productivo con el semillero? La cualificación del tutor no debe ser solo posgradual sino también pedagógica al igual que debe existir un real proceso investigativo en el grupo de investigación, ya que el eje de generación de conocimiento no debe girar en torno al semillero sino, por el 
contrario, el semillero debe nutrirse y crecer del proceso de los miembros del grupo. El tutor debe orientar, fomentar y modular a la realidad inmediata los intereses de los estudiantes, mas no imponer, coartar o interrumpir el proceso formativo con el fin de cumplir los objetivos del grupo y no del semillero.

Vale la pena aclarar que el proceso formativo de formulación investigativa es uno de los tantos procesos formativos del pregrado, y que la vinculación de las "semillas" a las actividades de un grupo de investigación es otro eje del proceso formativo; además, al ser parte de un grupo de investigación y participar en las líneas de investigación permite adquirir habilidades específicas y responsabilidades, que conlleva a entender las condiciones necesarias para consolidar un proceso investigativo hacia el futuro.

\section{El proceso formativo en investigación desde el semillero}

La existencia de semilleros de investigación es claramente una estrategia útil, pues a ellos acuden voluntariamente los estudiantes, motivados por el interés en un área específica del conocimiento, sobre la cual aspiran a aprender. Será necesario esperar más tiempo para saber si la estrategia funciona, porque lamentablemente por el momento no existen estudios que evalúen a fondo lo que ha significado para la Ciencia nacional la existencia de los semilleros de investigación y sus actividades formativas e investigativas $(9,14,19,20)$.

De otra parte, los semilleros también han sido utilizados como un medio para lograr objetivos a corto plazo como, por ejemplo, cuando los estudiantes de último año los utilizan para desarrollar sus trabajos de grado (21), excluyendo el proceso formativo, el cual es la parte más importante. Además, la vinculación de los estudiantes cuando ya están terminando su carrera no garantiza el relevo generacional pues permanecen muy poco tiempo en los semilleros; este relevo generacional es necesario para el cumplimiento de los procesos y la continuidad de los semilleros. Tampoco es correcta la participación en un semillero con la motivación dada por el afán de publicar y/o figurar, sin detenerse a revisar de forma rigurosa la calidad del proceso y los productos o experiencias que le permiten al estudiante desarrollar competencias suficientes para plantear una idea de investigación y evolucionarla hasta la obtención de resultados y productos idóneos $(1,22)$. Estos problemas no aportan al proceso, sino que lo dificultan.

\section{Las prioridades gubernamentales y el desarrollo investigativo juvenil}

En general, todos los gobiernos tienen planteadas políticas que buscan incentivar el talento de jóvenes inquietos por la ciencia(23), aunque la mayoría de las veces se quedan solo en el papel. En Colombia, la viabilidad y competitividad de proyectos de alto impacto y de equipos interdisciplinares dedicados a la búsqueda de soluciones a problemáticas específicas se ve reducida ante el pobre aporte de recursos (17), pues el presupuesto destinado a la investigación en la última década ha disminuido, pero, a pesar de ello la producción de artículos científicos Médicos se mantiene e incluso se ha incrementado (Figura 1). El año pasado se realizó un recorte drástico e injustificado al presupuesto

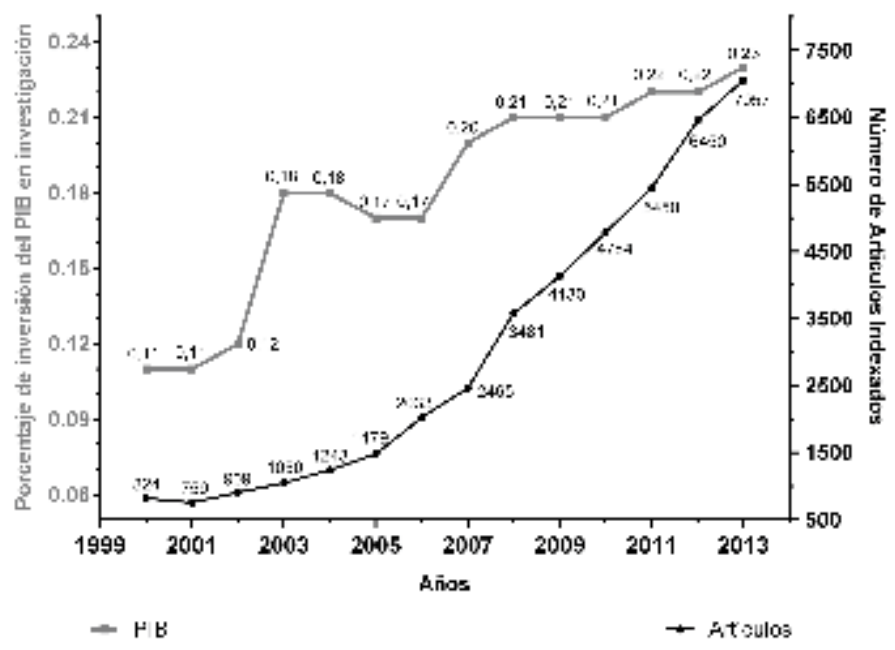

Fuente: World Bank. 2016. Revisado Abril 07, 2016, enlace: http://data. worldbank.org/indicator.

Fuente: SCImago. (2007). SJR - SCImago Journal \& Country Rank. Revisado abril 07, 2016, enlace: http://www.scimagojr.com

Figura 1. Comparación entre la inversión en investigación científica y la publicación en revistas indexadas en el área de medicina desde el año 2000 a 2013 en Colombia. 
destinado para el fondo nacional de investigaciones del país, lo que corresponde a $0.21 \%$ del Producto Interno Bruto (PIB), muy poco en comparación con otros países como Chile $(0.36 \%)$, Brasil $(1.15 \%)$, México $(0.43 \%)$ o Estados Unidos $(2.81 \%)$ que destinan este porcentaje anual anotado previamente, según el Banco Mundial.

En 1998, Colciencias decidió implementar un sistema de clasificación para los distintos centros de investigación del país, con el propósito de generar políticas de financiación, dejando a un lado la evidente necesidad de llevar la educación basada en el método tradicionalista a uno donde la cultura investigativa fuese el nuevo referente de la universidad (24); sin embargo, este modelo de medición de grupos deja de lado la formación del investigador, pues se limita solamente a los resultados y no hace un seguimiento del proceso.

La figura académica de la investigación en el país se inició con la conformación de Colciencias en 1968. El Decreto 80 de 1980 involucró el componente investigativo en el currículum Universitario; para la misma década se implementaron como requisitos la realización de tesis $\mathrm{y}$ trabajos de grado y posteriormente se creó el Consejo Nacional de Ciencia y Tecnología en 1990. Además, La ley 30 de 1992 establece en su artículo 20: "la denominación de universidad se otorga a aquellas instituciones que demuestren en la acreditación experiencia en investigación científica de alta calidad"; $y$ en 1994 se creó una comisión de ciencia, desarrollo y tecnología denominada la "misión de los sabios", la cual estableció la necesidad de incentivar la investigación en el país, debido a que las actividades de corte investigativo estaban atrasadas respecto a las desarrolladas por otros países $(2,17,25)$.

\section{Historia de los semilleros en Colombia}

En 1993 nace en la Universidad de Antioquia una iniciativa de investigación desde estudiantes de pregrado, que se denominó "semillero" (por su analogía con la agricultura), la cual dos años después es conocida por docentes y estudiantes de la Universidad del Cauca, quienes acogen la estrategia y la replican. A pesar de las dificultades para iniciar, algunos grupos de distintas universidades del país se congregaron en la ciudad de Manizales donde realizaron su primer intercambio de experiencias en el año de 1997 (20); posteriormente, en el Tercer Encuentro de Semilleros de Investigación realizado en el año 2000 en Popayán, se consolidó la Red Colombiana de Semilleros de Investigación (RedCOLSI). Desde hace cuatro años existe una red alterna que agrupa los semilleros de las Universidades del eje Cafetero, norte del Valle y Chocó llamada Red Regional de Semilleros de Investigación (Rredsi), cuyas instituciones no hacen parte de la RedCOLSI.

Más tarde, en el marco del segundo encuentro nacional de semilleros de investigación realizado a finales de julio de 2013, se conformó la Red Nacional de Semilleros de
Investigación de Ciencias de la Salud (RENASIS) como un espacio de intercambio académico y trabajo colectivo futuro entre los semilleros de Colombia.

Por otro lado, están las asociaciones Médicas estudiantiles (algunas de las cuales llevan más de 20 años de existencia) (26), que por medio de sus comités científicos desarrollan actividades de formulación y ejecución de proyectos comunitarios.

\section{Reflexión}

Lo anterior evidencia que en nuestro país existen espacios que permiten y fomentan el desarrollo de una cultura y disciplina investigativa, comprendiendo el proceso como una oportunidad del estudiante para formular cuestionamientos propios de su formación profesional, conociendo distintas facetas de esta. Este proceso se convierte en una ventaja para la realización de estudios ulteriores, dando un perfil más cualificado de los aspirantes a estudios de posgrado, considerando que desarrollaron competencias durante su pregrado $(1,9)$.

El ambiente que propician los semilleros es el ideal para guiar las destrezas de algunos jóvenes inquietos hacia una enseñanza y aprendizaje adecuados que realmente logre los objetivos de la formación investigativa $(6,11,20)$, cumpliendo a cabalidad el deber ser del investigador, sin necesidad de convertirlo en un proceso desagradable y dispendioso.

\section{Conclusiones}

Los semilleros de investigación son un espacio para la formación de estudiantes universitarios en áreas como el trabajo colaborativo y donde se desarrollan destrezas en organización, redacción de textos, habilidades de laboratorio, entre otras, todas ellas fundamentales para la formación integral de los estudiantes.

Además, el espacio pedagógico de los semilleros permite el desarrollo personal poniendo a prueba las habilidades analíticas, creativas y propositivas de los estudiantes; lo cual permite forjar un ser integral y brindar las herramientas para hallar soluciones a los problemas que afronte en su futura práctica profesional.

Es necesario que las universidades del país incentiven en sus estudiantes la importancia de la investigación, como parte de un proceso de formación integral en el estudiante de Medicina.

La generación de redes de investigación entre semilleros son un espacio de intercambio de metodologías de formación en investigación, conocimientos y generación de proyectos colaborativos que entes gubernamentales y las universidades del país deben apoyar e incentivar. 


\section{Referencias}

1. Fischer MR. Undergraduate Medical Education as a Foundation for Health Care and Research. Rev Dtsch Arztebl Int 2012; 109(18): 325-326.

2. Silva TDN, da Cunha Aguiar LC, Leta J, Santos DO, Cardoso FS, Cabral LM, et al. Role of the undergraduate student research assistant in the new millennium. Rev Cell Biol Educ. 2004; 3(4): 235-40.

3. Salgueira A, Costa P, Gonçalves M, Magalhães E, Costa MJ. Individual characteristics and student's engagement in scientific research: a cross-sectional study. Rev BMC Medical Education 2012; 12: 95.e1-9.

4. Eraso F, Narváez J, Lagos C, Escobar E, Erazo O. Aprendizaje significativo por investigación: propuesta alternativa. Rev. Cient 2014; 2 (19): 158-167.

5. Villar NM, González MFC, León MI, Cortés MC. La política científica en la formación de profesionales en las ciencias médicas. Una mirada reflexiva. Rev Medisur. 2014; 12(1): 310-314.

6. Huicho L. La medicina basada en evidencias como un nuevo paradigma de la enseñanza y la práctica de la medicina. RevAn. Fac. med. 1997; 58(2): 118-127.

7. Hunskaar S, Breivik J, Siebke M, Tømmerås K, Figenschau K, Hansen J-B. Evaluation of the medical student research programme in Norwegian medical schools. A survey of students and supervisors. BMC Medical Education. 2009; 9(1): 43.e1-8.

8. de Oliveira NA, Luz MR, Saraiva RM, Alves LA. Student views of research training programmes in medical schools. Rev Med Educ. 2011;45(7):748-55.

9. González J. Semilleros de Investigación: una estrategia formativa. Psychologia. Avances de la disciplina. 2008; 2(2):185-90.

10. Jacobs CD, Cross PC. The value of medical student research: the experience at Stanford University School of Medicine. Rev Med Educ. 1995; 29(5):342-6.

11. Solomon SS, Tom SC, Pichert J, Wasserman D, Powers AC. Impact of medical student research in the development of physician-scientists. Rev J Investig Med. 2003; 51(3):149-56.

12. Cádiz J, Villanueva OL, Echenique MJ, Astorga ML. ¿Profesores competentes o humanizadores? . Rev educ.educ. 2012; 15(3): 535-546.

13. Machado JE, Machado ME. The Role of Research Incubators in Encouraging Research and Publication Among Medical Students. Rev Acad Med. 2014; 89(7): 961-2.
14. Quintero J, Molina AM, Munévar FI. Semilleros de investigación: una estrategia para la formación de investigadores. Rev educ.educ. 2008; 11(1):31-42.

15. Taype Á, Bazán S, Valladares D. Producción Científica de las sociedades Científicas de estudiantes de medicina del Perú. 2002-2012. Rev CIMEL 2014; 18(1) 23-29.

16. Rose S. Publishing undergraduate research: creating opportunities to enhance the student learning experience within a research-intensive university. Rev Inspiring Academic Practice. 2014; 1(2): e1-9.

17. Crespi G, Dutrénit G. Science, Technology and Innovation Policies for Development. New York: Ed Springer; 2014: 1-14.

18. Prunuske AJ, Wilson J, Walls M, Clarke B. Experiences of mentors training underrepresented undergraduates in the research laboratory. Rev CBE Life Sci Educ. 2013; 12(3): 403-9.

19. González SV, Oquendo Puerta SR, Castañeda Gómez B. Semilleros de investigación. Editorial Biogénesis. 2010.

20. Oquendo S, González S, Castañeda B. Semilleros de Investigación: una emergencia en pos del conocimiento y la ciudadanía. REDSIN-Red Semilleros de Investigación de la Universidad de Antioquia, Medellín. 2005.

21. Uribe JI, Márquez CV, Amador G, Chavez AM. Percepción de la investigación científica e intención de elaborar tesis en estudiantes de psicología y enfermería. Enseñanza e investigación en psicología 2011; 16 (1): 15-26.

22. Al-Halabi B, Marwan Y, Hasan M, Alkhadhari S. Extracurricular research activities among senior medical students in Kuwait: experiences, attitudes, and barriers. Rev Adv Med Educ Pract 2014; 5: 95-101.

23. Richards R, Lewis R, Manoylov K, Brown R, Busch H. Our Journey Towards Institutionalizing Undergraduate Research.Transformative Dialogues: Teaching \& Learning Journal 2014; 7(1): e1-10.

24. Leon-Sarmiento F, Bayona-Prieto J, Bayona E, León M. Colciencias e inconciencias con los científicos colombianos: de la edad de piedra al factor de impacto. Rev Sal Pub 2005; 7: 227-35.

25. González JAL. Investigación y Universidad. ZOOCIENCIA. 2014; 1(1): 2-3.

26. Tristán PM. Sociedades científicas de estudiantes de medicina: el futuro de la investigación en Latinoamérica. MedUNAB 2010;13(1): 3-4. 